\title{
OC24 - An algorithm proposal to oral feeding in premature infants
}

\author{
Florbela Neto (Portugal) ${ }^{1}$; Ana Paula França (Portugal)2; \\ Sandra Cruz (Portugal) $)^{2}$
}

${ }^{1}$ ICBAS-Instituto de Ciencias Biomédicas Abel Salazar; ${ }^{2}$ ESEP-Escola Superior de Enfermagem do Porto

Theme: Transition of care.

Keywords: nursing interventions, premature feeding skills, sucking, swallowing.

Introduction: Oral feeding is one of the hardest steps for premature infants after respiratory independence and is a challenge for nurses in neonatology.

Objectives: To know the characteristics of preterm infants, essential for oral feeding; to know the nurses' opinion on nursing interventions, that promote the transition of gavage feeding for oral feeding in preterm infants.

Methods: An exploratory, descriptive study with a qualitative approach was used.

Semi-structured interviews with neonatal nurses were conducted and data was submitted to content analysis.

Results: Weight, gestational age, physiological stability, sucking coordination, swallowing and breathing, and the overall look and feeding involvement are fundamental parameters to begin oral feeding. Positioning the baby, reflexes stimulation, control stress levels, monitoring the temperature and the milk flow are nursing interventions that promote the development of feeding skills.

Conclusion: An algorithm for the oral feeding of preterm infants was developed grounded in the opinions of nurses.

\section{OC25 - Helping parents spot the deteriorating child: co-production of safety-netting interventions}

Sarah Neill (United Kingdom) ${ }^{1 ;}$, Damian Roland (United Kingdom)2;

Matthew Thompson (United States of America);

Juliet Usher-Smith (United Kingdom) ${ }^{4}$; Laura Mullins

(United Kingdom); ; Monica Lakhanpaul (United Kingdom) ${ }^{6}$

${ }^{1}$ University of Northampton; ${ }^{2}$ University of Leicester and Univeristy Hospitals Leicester; ${ }^{3}$ University of Washington; ${ }^{4}$ University of Cambridge; ${ }^{5}$ Lay member;

${ }^{6}$ University College London

Theme: Child protection and managing risk.

Keywords: Acute illness, co-production, patient safety, safety netting. 
Introduction: Providing families with a safety net of information is a crucial component in the care of children with acute illnesses at home (Jones et al 2013). Safety netting is recommended by UK national bodies and has been found to reduce re-consultation safely (Maguire et al 2011).

Aim: To provide parents with information to help them determine when to seek help for an acutely ill child.

Methods: Research programme encompassed five projects: a systematic review; a qualitative exploration of safety netting information used by parents and professionals; a mixed methods development of content, format and delivery modes for the first intervention; video capture of children with acute illness; and co-design of the content of the intervention with parents and professionals.

Conclusion: ASK SNIFF projects repeatedly demonstrate the need for professionally endorsed, co-produced safety-netting resources for parents with varying information needs, literacy levels and ability to use information technology.

\title{
OC26 - Parents of young children in research: informants, consultants and collaborators
}

\author{
Sarah Neill (United Kingdom) ${ }^{1}$; Damian Roland (United Kingdom)2; \\ Matthew Thompson (United States of America); ${ }^{3}$ Natasha Bayes \\ (United Kingdom) $)^{1}$; Laura Mullins (United Kingdom) ${ }^{4}$; \\ Monica Lakhanpaul (United Kingdom) ${ }^{5}$
}

${ }^{1}$ University of Northampton; ${ }^{2}$ University of Leicester and University Hospitals Leicester; ${ }^{3}$ University of Washington; ${ }^{4}$ Lay member; ${ }^{5}$ University College London

Theme: Ethical issues: dignity and humanity.

Keywords: Parents, research participation; research involvement; research collaboration.

Introduction: Patient and public involvement in research is recognised as best practice in the UK, as services developed with service users are more likely to meet their needs. In child health this often means engaging with parents of young children.

Aim: To share experiences of engaging with parents of young children in research projects at varying levels of participation.

Methods: Methods include parents as participants, as parent panels and as research team members reflecting various steps on the participation ladder (Hart 1992).

Results: Parents report personal benefits and a range of different challenges when engaging in child health research which impact the management/delivery of research projects.

Conclusion: Key elements of working with parents include time to plan involvement, building respect/mutual understanding, equal access to project activities, continual support for parents, reward and recognition. Research projects need to cost and plan for parents' engagement in research for it to be successful. 